\title{
ORIGINAL
}

\section{VARIABILIDAD EN LA UTILIZACIÓN DE NUEVOS MEDICAMENTOS EN UN ÁREA DE ATENCIÓN PRIMARIA}

\author{
Ma Jesús Lallana Álvarez (1) y Ma José Rabanaque Hernández (2) \\ (1) Dirección de Atención Primaria Sector Zaragoza III. Centro de Salud Seminario. \\ (2) Departamento de Medicina Preventiva y Salud Pública. Universidad de Zaragoza.
}

\begin{abstract}
RESUMEN
Fundamento: La utilización de nuevos fármacos ha aumentado de forma importante en los últimos años. El objetivo de este trabajo es conocer la utilización de novedades farmacéuticas en un área de salud de Zaragoza, describir cuales son las más utilizadas y su evolución en el tiempo y analizar las características de los centros que se asocian con un mayor uso de estos medicamentos.
\end{abstract}

Métodos: Se trata de un estudio descriptivo en el que se analizaron todas las dispensaciones de 50 nuevos principios activos comercializados entre el 1-1-2000 y el 31-12-2003 en un área de salud. Se calculó el porcentaje de utilización de novedades durante el primer año de su comercialización, en cada centro de atención primaria, y se analizaron las características de los centros que se asociaban con mayor grado de innovación.

Resultados: El 76\% de los 50 nuevos fármacos comercializados eran escasamente innovadores respecto a los ya existentes. Las diez novedades más prescritas comenzaron a utilizarse en el primer cuatrimestre tras su aparición en el mercado. El porcentaje de utilización de novedades respecto al total de fármacos prescritos en los centros de salud estudiados osciló entre el 0,3\% y el 1,18\%. En el análisis efectuado por centros, el «gasto por paciente» y las «consultas por médico» se correlacionaron positivamente con un mayor uso de medicamentos nuevos, mientras que el porcentaje de pensionistas presentó una relación inversa.

Conclusiones: Existe una gran variabilidad en el porcentaje de utilización de novedades farmacológicas entre los centros de atención primaria. Los centros con mayor número de consultas por facultativo son los que tienden a incorporar los nuevos medicamentos con mayor facilidad.

Palabras clave: Utilización de medicamentos. Atención primaria de salud.

Correspondencia:

Ma Jesús Lallana Álvarez

Dirección de Atención Primaria Sector Zaragoza III.

Centro de Salud Seminario

C/Condes de Aragón, 30

50009 Zaragoza

Correo electrónico: mjlallana@ salud.aragon.es

\section{ABSTRACT \\ Variability in the Use of New Drugs in a Primary Care District}

Background: The use of new drugs has undergone a major increase in recent years. This study is aimed at ascertaining the degree to which pharmaceutical novelties are being used in one healthcare district in Zaragoza, describing which are those most used and the trend thereof over the course of time in addition to analyzing the characteristics of those centers associated with a greater use of these drugs.

Methods: A descriptive study analyzing all of the dispensations of fifty new active ingredients marketed within the January 1, 2000 December 31, 2003 period in one healthcare district. The percentage of use of novelties throughout the first year these drugs were marketed at each primary care center was calculated, the characteristics of those centers associated with a greater degree of innovation having been analyzed.

Results: Seventy-six percent of the fifty new drugs marketed were barely innovative in comparison to the existing ones. The ten new drugs most prescribed began being used during the first four months immediately after coming out on the market. The percentage of use of new drugs as compared to all of the drugs prescribed at the healthcare centers studied fell within the $0.3 \%-1.18 \%$ range. In the analysis made by centers, the «per-patient expense» and the «consultations per physician» are positively correlated with a greater use of new drugs, whilst the percentage of retirees showed a reverse ratio.

Conclusions: A high degree of variability exists in the percentage of use of pharmaceutical novelties among the primary care centers. The centers having the largest number of consultations per physician are those tending toward more readily incorporating these new drugs.

Key words: Drug utilization. Primary health care. 


\section{INTRODUCCIÓN}

El elevado número de medicamentos que se introducen anualmente en el mercado farmacéutico, y la amplia utilización de los mismos, puede tener importantes implicaciones clínicas y económicas para los sistemas sanitarios ${ }^{1-3}$.

Se ha descrito que la mayoría de los medicamentos autorizados en los últimos años en España son poco innovadores, aportando ventajas que no son relevantes frente a los ya existentes (son los llamados medicamentos «me too»). Esto ocurre también en otros países; en un informe que analiza todos los nuevos fármacos incorporados al mercado estadounidense durante el periodo 1989-2000, de un total de 1.035 sólo 153 (15\%) podían considerarse fármacos nuevos con una aportación clínica significativa ${ }^{3}$.

También se ha observado un aumento espectacular en la comercialización de «formas modificadas» de medicamentos conocidos. Dentro de esta categoría se incluyen las formas farmacéuticas de liberación modificada, los estereoisómeros, los metabolitos activos etc. cuyos porcentajes de prescripción están alcanzando cuotas bastante elevadas ${ }^{3-5}$.

La decisión de prescribir un nuevo medicamento conlleva repercusiones clínicas y terapéuticas de gran trascendencia dada la limitada información que existe sobre su eficacia y seguridad a largo plazo en las condiciones reales de uso. Por otro lado, el retraso innecesario en la prescripción podría privar al paciente de un posible beneficio ${ }^{6,7}$.

Aunque los estudios sobre la prescripción de nuevos fármacos son escasos sus resultados reflejan un crecimiento en la utilización de las llamadas novedades que no se corresponde con su eficacia real ${ }^{8}$, presentando además una gran variabilidad -entre países, regiones, centros e incluso individuos- no explicable tan sólo por las diferencias en la morbilidad de las poblaciones. En un estudio se observó que un $10 \%$ de los médicos prescribía el $40 \%$ de los medicamentos nuevos ${ }^{7}$. También se ha observado que los médicos que prescriben antes los medicamentos nuevos parecen tener un gran número de pacientes en su consulta diaria y confían más en las fuentes comerciales de información ${ }^{9}$.

El objetivo de este trabajo es conocer la utilización de novedades farmacéuticas en un área de salud de Zaragoza, describir cuáles son las más utilizadas y su evolución en el tiempo, y analizar las características de los centros que se asocian con un mayor uso de estos medicamentos.

\section{SUJETOS Y MÉTODOS}

El estudio se llevó a cabo en todos los centros de Atención Primaria del área 3 de Zaragoza y recoge datos del periodo 2000-2003. En el momento del estudio al área de salud 3 pertenecían 32 centros de salud que en el año 2003 atendían a una población de 313.321 habitantes. Se analizaron todas las dispensaciones que se realizaron desde el 1-1-2000 hasta el 31-12-2003, a partir de las recetas prescritas por los médicos. Estas recetas son mecanizadas mensualmente por el Colegio Oficial de Farmacéuticos y la información es procesada por el Servicio de Inspección Farmacéutica que proporciona los datos correspondientes a cada área de salud de la Comunidad Autónoma de Aragón. En cada área se tratan los datos con un programa específico que permite asignar el principio activo a cada especialidad farmacéutica y calcular el consumo agregado por principios activos y por centros de salud.

Los principios activos seleccionados fueron aquellos comercializados por primera vez en España entre el 1-1-2000 y el 31-122003. Para identificarlos se extrajeron del Nomenclator oficial de productos farmacéuticos por su fecha de alta. Se incluyeron en el estudio aquellos fármacos que se utilizan en 
Atención Primaria, excluyendo los calificados como de Diagnóstico Hospitalario (DH) o uso Hospitalario (H). También se excluyeron las asociaciones de varios medicamentos si éstos ya estaban comercializados anteriormente.

Cada medicamento se clasificó según su valor terapéutico en base a los criterios fijados por la revista Información Terapéutica del Sistema Nacional de la Salud publicada por el Ministerio de Sanidad y Consumo, en la que se comparan los nuevos medicamentos con el resto de los existentes en España en el momento de su autorización para conocer su valor relativo dentro del arsenal terapéutico ${ }^{10}$.

En el estudio se realizó primero un análisis por fármaco en el que se determinó el número de envases prescritos de cada principio activo seleccionado durante un periodo de un año, contado a partir de la fecha en la que apareció la primera receta dispensada.

En segundo lugar, se procedió a un análisis por centro de salud. Se contabilizaron los envases de nuevos medicamentos prescritos por cada centro durante el primer año de su comercialización y se compararon con el número de envases totales prescritos en el centro durante el periodo de estudio, calculando el porcentaje de innovación.

Para analizar su posible relación con el porcentaje de innovación para cada centro se recogieron las siguientes variables:

- Tipo de centro: urbano y rural con más de 10.000 habitantes y rural con menos de 10.000 habitantes.

- Años que lleva funcionando como equipo de Atención Primaria.

- Número de médicos por equipo.

- Población y porcentaje de pensionistas asignados a cada centro, según la información que se obtiene de la base de datos de Tarjeta Sanitaria.
- Gasto farmacéutico mensual por persona protegida (GPP), que se calculó dividiendo el gasto farmacéutico de activos más el de pensionistas por la población total (activos más pensionistas) y por el número de meses considerados.

- Datos de actividad del centro. Los datos de actividad de los años 2000 y 2001 de los centros del área 3 estaban editados en las memorias anuales del área. Se obtuvieron de esta fuente los datos sobre número de consultas por profesional y frecuentación. Los datos de 2002 y 2003 se obtuvieron del Sistema de Información de la Gerencia sobre actividad profesional. Son datos que los centros remiten mensualmente a la Gerencia sobre la actividad realizada.

El análisis de los datos se realizó con el programa estadístico SPSS. En la comparación de las medias de variables cuantitativas se utilizó el método ANOVA, cuando existía normalidad en la distribución e igualdad de varianzas. Cuando no se cumplieron estos criterios se aplicó el test de Kruskal-Wallis. La relación entre variables cuantitativas se realizó mediante pruebas de correlación. En las comparaciones se ha fijado un nivel de confianza del $95 \%$, aceptándose como significativas las diferencias encontradas cuando el valor de $\mathrm{p}$ fue inferior a 0,05 .

Tomando como unidad de análisis los centros de salud, mediante modelos de regresión lineal múltiple, se analizó la relación entre el porcentaje de novedades sobre envases totales y las variables gasto farmacéutico por paciente, número de consultas por facultativo, porcentaje de pensionistas, tasas de frecuentación por centro de salud y años de funcionamiento del centro como modelo reformado de atención primaria. Se utilizó el método por pasos hacia delante, incluyendo las variables con probabilidad $\mathrm{F}$ superior o igual a 0,05 y excluyendo las que presentaban valores superiores o iguales a 0,10 . 
Tabla 1

Características de los fármacos incluidos en el estudio

\begin{tabular}{|c|c|c|c|c|c|}
\hline grupo terapeutico & principio activo & $\begin{array}{c}\text { potencial } \\
\text { terapeutico }\end{array}$ & año comercialización & $\begin{array}{c}\text { envases } \\
\text { prescritos el } \\
\text { primer año } \\
\$\end{array}$ & $\begin{array}{l}\text { precio medio } \\
\text { por envase }\end{array}$ \\
\hline \multirow{8}{*}{$\begin{array}{l}\text { A: aparato digestivo y } \\
\text { metabolismo }\end{array}$} & dosmalfato & $\mathrm{C}$ & 2000 & 696 & $18,6 €$ \\
\hline & esomeprazol & $\mathrm{C}$ & 2002 & 2835 & $19,2 €$ \\
\hline & levosulpirida & $\mathrm{C}$ & 2002 & 1238 & $7,2 €$ \\
\hline & racecadotrilo & $\mathrm{C}$ & 2002 & 433 & $8,9 €$ \\
\hline & insulina aspara & $\mathrm{C}$ & 2002 & 812 & $99,5 €$ \\
\hline & rosiglitazona & $\mathrm{B}$ & 2001 & 198 & $53,0 €$ \\
\hline & nateglinida & $\mathrm{C}$ & 2002 & 254 & $38,4 €$ \\
\hline & pioglitazona & $\mathrm{C}$ & 2002 & 209 & $62,1 €$ \\
\hline B: sangre & fondaparinux & $\mathrm{B}$ & 2003 & $23 \dagger$ & $141,5 €$ \\
\hline \multirow{3}{*}{ C: cardiovascular } & barnidipino & $\mathrm{C}$ & 2001 & 537 & $19,2 €$ \\
\hline & eprosartan & $\mathrm{C}$ & 2000 & 5436 & $25,3 €$ \\
\hline & manidipino & $\mathrm{C}$ & 2003 & $546+$ & $20,1 €$ \\
\hline $\begin{array}{l}\text { G: genitourinario y hormonas } \\
\text { sexuales }\end{array}$ & dienogest + estrageno & $\mathrm{C}$ & 2002 & 870 & $12,2 €$ \\
\hline \multirow[t]{3}{*}{$\mathrm{J}:$ antiinfecciosos } & telitromicina & $\mathrm{B}$ & 2002 & 1260 & $30,2 €$ \\
\hline & moxifloxacino & $\mathrm{C}$ & 2000 & 3216 & $25,7 €$ \\
\hline & brivudina & $\mathrm{C}$ & 2003 & $35 \dagger$ & $107,6 €$ \\
\hline L: antineoplásicos & exemestano & $\mathrm{C}$ & 2000 & 73 & $170,6 €$ \\
\hline \multirow[t]{6}{*}{ M: aparato locomotor } & rofecoxib & $\mathrm{B}$ & 2000 & 17191 & $38,6 €$ \\
\hline & celecoxib & $\mathrm{C}$ & 2000 & 13306 & $38,4 €$ \\
\hline & dexibuprofeno & $\mathrm{C}$ & 2001 & 12994 & $7,8 €$ \\
\hline & condroitinsulfato & $\mathrm{C}$ & 2003 & $2253 \dagger$ & $16,4 €$ \\
\hline & diacereina & $\mathrm{C}$ & 2003 & $1265 \dagger$ & $10,2 €$ \\
\hline & dexketoprofen & $\mathrm{C}$ & 2000 & 4106 & $7,1 €$ \\
\hline \multirow[t]{12}{*}{ N: sistema nervioso central } & fentanilo & $\mathrm{B}$ & 2001 & 372 & $48,3 €$ \\
\hline & buprenorfina & $\mathrm{C}$ & 2002 & 1678 & $46,4 €$ \\
\hline & almotriptan & $\mathrm{C}$ & 2000 & 563 & $34,9 €$ \\
\hline & eletriptan & $\mathrm{C}$ & 2003 & $3 \dagger$ & $12,8 €$ \\
\hline & oxcarbazepina & $\mathrm{B}$ & 2001 & 416 & $57,4 €$ \\
\hline & levetiracetam & $\mathrm{C}$ & 2002 & 536 & $104,7 €$ \\
\hline & cabergolina & $\mathrm{B}$ & 2002 & 674 & $51,2 €$ \\
\hline & quetiapina & $\mathrm{C}$ & 2000 & 882 & $41,1 €$ \\
\hline & amisulpride & $\mathrm{C}$ & 2002 & 81 & $112,0 €$ \\
\hline & ziprasidona & $\mathrm{C}$ & 2003 & $342 \dagger$ & $116,6 €$ \\
\hline & zaleplon & $\mathrm{C}$ & 2001 & 1474 & $5,1 €$ \\
\hline & pilocarpina & $\mathrm{D}$ & 2003 & 22 & $83,8 €$ \\
\hline P: antiparasitarios & permetrina & $\mathrm{A}$ & 2000 & 216 & $16,3 €$ \\
\hline \multirow[t]{4}{*}{ R: aparato respiratorio } & tiotropio bromuro & $\mathrm{B}$ & 2003 & $4931 \dagger$ & $53,9 €$ \\
\hline & desloratadina & $\mathrm{C}$ & 2002 & 6256 & $8,8 €$ \\
\hline & levocetirizina & $\mathrm{C}$ & 2003 & $476+$ & $7,2 €$ \\
\hline & rupatadina & $\mathrm{C}$ & 2003 & $889 \dagger$ & $9,0 €$ \\
\hline \multirow[t]{8}{*}{ S: órganos de los sentidos } & lomefloxacino & $\mathrm{C}$ & 2000 & 65 & $4,9 €$ \\
\hline & brinzolamida & $\mathrm{C}$ & 2000 & 2842 & $13,1 €$ \\
\hline & bimatoprost & $\mathrm{C}$ & 2002 & 1366 & $20,2 €$ \\
\hline & travoprost & $\mathrm{C}$ & 2002 & 1643 & $19,6 €$ \\
\hline & olopatadina & $\mathrm{C}$ & 2003 & $273+$ & $10,0 €$ \\
\hline & carmelosa & $\mathrm{D}$ & 2002 & 4578 & $5,9 €$ \\
\hline & emedastina & $\mathrm{C}$ & 2000 & 392 & $10,5 €$ \\
\hline & ketotifeno & $\mathrm{C}$ & 2001 & 324 & $9,4 €$ \\
\hline \multirow[t]{2}{*}{ V: varios } & sevelamer & $\mathrm{C}$ & 2001 & 82 & $147,2 €$ \\
\hline & risedronico acido & $\mathrm{B}$ & 2000 & 2837 & $42,1 €$ \\
\hline
\end{tabular}

*A: Importante mejora terapéutica (2\%); B: Modesta mejora terapéutica (18\%); C: Nula o muy pequeña mejora terapéutica (76\%); D: Sin clasificar (4\%). † Para los fármacos que se comercializaron en 2003, no ha podido completarse un año de seguimiento y el número de envases que se contabiliza desde que apareció la primera receta hasta el 31 de diciembre de 2003. \$ Primer año tras su comercialización 
Tabla 2

Coste de las 15 novedades más prescritas y de los fármacos de referencia dentro de su grupo terapéutico

\begin{tabular}{|l|c|c|c|}
\hline Nuevos fármacos & coste tratamiento día* & fármaco de referencia & $\begin{array}{c}\text { Coste tratamiento día del } \\
\text { fármaco de referencia }\end{array}$ \\
\hline Rofecoxib & 1,35 & ibuprofeno, diclofenaco & 0,3 \\
\hline Delecoxib & 1,34 & ibuprofeno, diclofenaco & 0,3 \\
\hline Dexibuprofeno & 0,75 & ibuprofeno & 0,32 \\
\hline Cesloratadina & 0,62 & loratadina & 0,92 \\
\hline Eprosartan & 0,92 & losartan, irbesartan & 0,5 \\
\hline Tiotropio bromuro & 1,91 & ipratropio & 0,15 \\
\hline Carmelosa & 0,60 & alcohol polivinilico, polividona & 3,4 \\
\hline Dexketoprofen gel & 7,77 & diclofenaco gel & 4,38 \\
\hline Moxifloxacino & 4,92 & levofloxacino & 0,44 \\
\hline Brinzolamida & 0,46 & dorzolamida & 1,44 \\
\hline Risedronico acido & 1,49 & alendronato & 0,36 \\
\hline Esomeprazol & 1,48 & omeprazol & 0,34 \\
\hline Condroitinsulfato & 0,7 & naproxeno & 2,92 \\
\hline Buprenorfina & 2,92 & fentanilo parches & \\
\hline Travoprost & 0,73 & & \\
\hline
\end{tabular}

* El Coste Tratamiento Día (CTD) se ha calculado como el coste en euros de una dosis diaria definida (DDD) del fármaco a partir del precio del envase más económico cuya unidad de contenido corresponde con una DDD. En el caso de la Carmelosa y la polividona, el CTD se ha calculado considerando una posología de 3 gotas en cada ojo al día. Para el dexketoprofen y el diclofenaco gel se ha tomado como CTD el precio total del envase de 60 gramos.

\section{RESULTADOS}

Durante los años 2000-2003 se comercializaron 50 nuevos principios activos de uso potencial en Atención Primaria, correspondientes a 12 grupos terapéuticos cuyas características se recogen en la tabla 1.

De los nuevos fármacos 38 (76\%) no suponían una mejora terapéutica respecto a los ya existentes, en cambio representaban un importante porcentaje sobre el total de envases de novedades prescritas: 71.281 $(68,5 \%)$. No se encontraron diferencias significativas en el número de envases prescritos según el valor terapéutico (KruskalWallis $\mathrm{chi}^{2}=1,101 ; \mathrm{p}=0,777$ ).
Para analizar el coste de los nuevos medicamentos se eligieron los 15 fármacos que suponían el $80 \%$ de la prescripción de novedades en número de envases el primer año tras su puesta en el mercado (tabla 2) y se compararon con los fármacos de referencia dentro de su grupo terapéutico. El coste/tratamiento/día (CTD) de los nuevos fármacos fue 4 veces más alto para 5 de esos 15 fármacos (rofecoxib, celecoxib, tiotropio, carmelosa y esomeprazol) y para otros 4 fue más del doble (dexibuprofeno, desloratadina, dexketoprofeno y condroitinsulfato). Para los 6 fármacos restantes el coste era similar al de los fármacos con los que se comparaban. 


\section{Figura 1}

Número de centros que prescriben una novedad terapéutica en el primer año de su comercialización

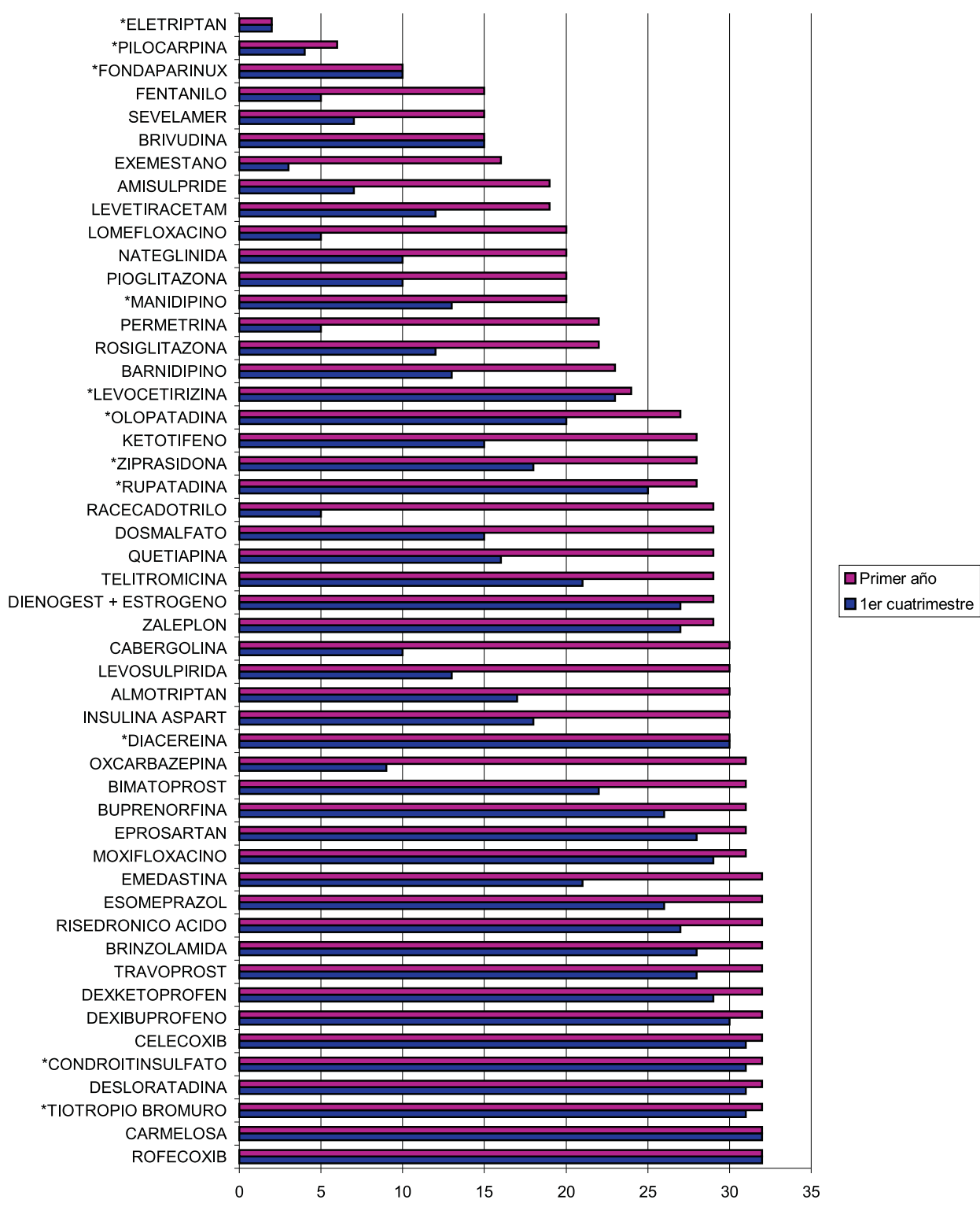

* Fármacos que se comercializaron en 2003, para los que no ha podido completarse un año de seguimiento

En la figura 1 se presenta el número de centros que prescribe una novedad terapéutica en su primer año en el mercado.
Hay 13 fármacos prescritos por todos los centros del área, y 12 de ellos fueron prescritos por más del $80 \%$ de los centros en el 
Tabla 3

Porcentaje de utilización de las novedades en cada centro de Atención Primaria

\begin{tabular}{|c|c|c|c|c|c|c|}
\hline Centro & $\begin{array}{c}\mathbf{N}^{0} \text { diferentes } \\
\text { novedades }\end{array}$ & Tipo de Centro* & $\%$ novedades $\dagger$ & $\begin{array}{l}\text { Media } \\
\text { GPP \$ }\end{array}$ & $\mathbf{N}^{0}$ Médicos & $\begin{array}{c}\text { \% envases sobre el total de } \\
\text { novedades de los } 15 \text { fármacos } \\
\text { nuevos más prescritos } \ddagger\end{array}$ \\
\hline 1 & 50 & 1 & 0,61 & $14,64 €$ & 14 & 85,48 \\
\hline 2 & 49 & 2 & 0,72 & $19,36 €$ & 11 & 80,03 \\
\hline 3 & 48 & 2 & 0,70 & $15,65 €$ & 7 & 83,33 \\
\hline 4 & 48 & 1 & 0,57 & $13,16 €$ & 16 & 83,72 \\
\hline 5 & 47 & 2 & 0,58 & $18,52 €$ & 12 & 86,93 \\
\hline 6 & 46 & 1 & 0,77 & $16,81 €$ & 14 & 81,53 \\
\hline 7 & 45 & 2 & 0,68 & $19,07 €$ & 14 & 84,99 \\
\hline 8 & 45 & 1 & 0,84 & $15,64 €$ & 8 & 86,57 \\
\hline 9 & 44 & 3 & 0,68 & $17,07 €$ & 12 & 84,63 \\
\hline 10 & 44 & 3 & 0,96 & $19,02 €$ & 6 & 87,04 \\
\hline 11 & 44 & 2 & 0,82 & $16,20 €$ & 7 & 88,60 \\
\hline 12 & 44 & 2 & 0,97 & $15,51 €$ & 9 & 85,82 \\
\hline 13 & 44 & 1 & 0,98 & $14,26 €$ & 9 & 82,77 \\
\hline 14 & 44 & 1 & 0,70 & $14,61 €$ & 5 & 83,12 \\
\hline 15 & 43 & 3 & 1,12 & $19,25 €$ & 7 & 90,32 \\
\hline 16 & 43 & 3 & 0,95 & $17,88 €$ & 6 & 88,17 \\
\hline 17 & 43 & 2 & 0,61 & $13,89 €$ & 9 & 87,96 \\
\hline 18 & 43 & 2 & 1,18 & $13,10 €$ & 5 & 90,09 \\
\hline 19 & 42 & 3 & 0,71 & $16,41 €$ & 7 & 86,24 \\
\hline 20 & 41 & 3 & 1,09 & $18,18 €$ & 5 & 90,84 \\
\hline 21 & 41 & 3 & 1,14 & $29,20 €$ & 7 & 90,86 \\
\hline 22 & 40 & 3 & 0,85 & $20,74 €$ & 8 & 89,08 \\
\hline 23 & 39 & 3 & 0,74 & $22,50 €$ & 5 & 84,71 \\
\hline 24 & 39 & 3 & 0,66 & $16,73 €$ & 10 & 83,21 \\
\hline 25 & 38 & 3 & 0,64 & $24,38 €$ & 9 & 92,57 \\
\hline 26 & 38 & 3 & 0,98 & $24,20 €$ & 5 & 92,30 \\
\hline 27 & 36 & 1 & 0,61 & $10,15 €$ & 4 & 90,61 \\
\hline 28 & 35 & 3 & 0,63 & $21,35 €$ & 5 & 89,71 \\
\hline 29 & 32 & 3 & 0,47 & $17,60 €$ & 6 & 89,41 \\
\hline 30 & 30 & 3 & 0,79 & $22,42 €$ & 4 & 95,40 \\
\hline 31 & 29 & 3 & 0,69 & $24,08 €$ & 4 & 91,97 \\
\hline 32 & 22 & 3 & 0,30 & $14,61 €$ & 4 & 95,43 \\
\hline
\end{tabular}

*1: Centros en Zaragoza capital; 2: Centros rurales, población >10.000 habitantes; 3:Centros rurales, población $<10.000$ habitantes. $\dagger$ El porcentaje de novedades se calcula $=(($ envases nuevos fármacos el primer año tras su comercialización)/(envases totales de fármacos prescritos $))^{*} 100$ \$ GPP: Gasto farmacéutico por persona protegida. $¥$ Para cada centro se calcula $=($ envases de los 15 fármacos nuevos más prescritos $) /($ envases de todos los fármacos nuevos prescritos).

primer cuatrimestre tras su comercialización.
Respecto al porcentaje de utilización de novedades por centro la media fue de $0,76 \%$ 
Tabla 4

Variables asociadas al porcentaje de novedades por centro de salud. Modelo de regresión lineal múltiple

\begin{tabular}{|l|c|c|c|}
\hline Variables incluidas en el modelo & $\beta$ & Significación & IC de $\beta$ al 95\% \\
\hline $\mathrm{N}^{\mathbf{o}}$ de consultas por facultativo & 0,005 & 0,088 & $-0,001-0,010$ \\
\hline Porcentaje de pensionistas & $-0,020$ & 0,006 & $-0,34-0,006$ \\
\hline Gasto farmacéutico por paciente & 0,057 & 0,000 & $0,031-0,084$ \\
\hline
\end{tabular}

Variables excluidas del modelo: tasa de frecuentación por centro de salud y años de funcionamiento como equipo de atención primaria. IC: intervalo de confianza.

con una variación entre el $0,30 \%$ y el $1,18 \%$ (tabla 3).

En la tabla 3 se muestra también la media del gasto farmacéutico por persona protegida en cada centro. Cuando se intentó relacionar con el porcentaje de novedades, realizando una correlación no paramétrica, no se observó relación significativa entre las dos variables $(\mathrm{p}=0,204)$.

El número de nuevos fármacos diferentes prescritos por cada centro aparece descrito en la tabla 3. Sólo un centro prescribió las 50 novedades estudiadas. Los centros rurales, con menos población y sólo 4 médicos en el equipo, son los que precribieron un menor número de novedades diferentes. El número de médicos por centro se relacionó de forma significativa con el número de nuevos principios activos diferentes prescritos $(\mathrm{r}=0,6518 ; \mathrm{p}=0,0005)$.

En todos los centros se observó que 15 nuevos fármacos suponían más del $80 \%$ del total de envases de novedades. En los 8 centros que prescribían menos fármacos nuevos diferentes (de 22 a 38 principios activos de un total de 50), el porcentaje que suponían los 15 fármacos más prescritos era del 89,41 al $95,43 \%$.

En el análisis multivariante realizado no se encontró asociación en ninguno de los modelos elaborados entre tasa de frecuentación y años de funcionamiento del centro como modelo reformado con el porcentaje de novedades. El modelo que explicaba mejor la variabilidad en el porcentaje de novedades $\left(r^{2}=0,521\right)$ es el que se presenta en la tabla 4. El número de consultas por facultativo y el gasto farmacéutico por paciente se asociaban con un mayor uso de medicamentos nuevos, mientras que el mayor porcentaje de pensionistas se asociaba de forma inversa a la variable analizada.

\section{DISCUSIÓN}

En el trabajo presentado se observa que los nuevos fármacos eran utilizados por los médicos de Atención Primaria en plazos de tiempo cortos tras su comercialización. Entre los resultados llama la atención que de los 50 fármacos analizados, únicamente 10 (20\%) aportaban alguna mejora respecto a lo que ya existía en el mercado. Algunos estu$\operatorname{dios}^{1,11}$ consideran muy importante en la adopción de novedades el tipo de fármaco y la percepción del riesgo que conlleva la utilización de los nuevos medicamentos, por lo que cabría esperar que aquellas novedades que aportan alguna mejora terapéutica fueran más prescritas que las que no suponen ningún avance. $\mathrm{Al}$ analizar el potencial terapéutico de los fármacos más prescritos en su primer año en el mercado (más de 2.000 envases), se observa que entre los 10 primeros sólo hay 2 (rofecoxib y bromuro de tiotropio) que fueron clasificados como innovadores. Como ocurre en otros estudios ${ }^{12-14}$ 
los fármacos clasificados como «novedades sin aportación terapéutica relevante» son prescritos en gran medida en el área estudiada. Esto parece sugerir que el médico no tiene especialmente en cuenta este criterio a la hora de introducir nuevos fármacos en su «vademecum particular».

Los porcentajes de utilización más elevados se dan en fármacos de grupos muy prescritos en la práctica habitual. Entre las 10 novedades más prescritas cuatro son antiinflamatorios no esteroideos (AINE) y pertenecen al grupo M (Aparato Locomotor). En el estudio de Tamblyn et $\mathrm{al}^{12}$ los mayores porcentajes de utilización de nuevos fármacos pertenecían a los grupos Sistema Nervioso Central (antidepresivos) y Aparato Digestivo y Metabolismo (antiulcerosos).

Los medicamentos que han sido más prescritos, tanto en número de envases como por mayor número de centros, lo han sido también de forma más rápida. Para las 10 novedades más prescritas en número de envases se inició la prescripción en el primer cuatrimestre tras su comercialización en más del $90 \%$ de los centros.

En lo que respecta al tiempo de adopción de las novedades, este se ve aumentado a mayor riesgo percibido para cada medicamento $^{11}$. Se puede argumentar que los 10 fármacos más prescritos (rofecoxib, celecoxib, dexibuprofeno, desloratadina, eprosartan, tiotropio, carmelosa, dexketoprofeno, moxifloxacino y brinzolamida) tenían un riesgo percibido bajo. Esto es debido a que 8 de ellos son fármacos considerados me too y su familiaridad con fármacos similares minimiza la percepción de sus riesgos ${ }^{1}$. No obstante, hay que tener en cuenta que cuando se comercializa un medicamento la información disponible sobre seguridad es limitada. Así por ejemplo, el rofecoxib se promocionaba confiando en su seguridad gastrointestinal. Posteriormente, el fármaco fue retirado del mercado por solicitud del laboratorio titular debido a los resultados de un ensayo clínico en el que se demostraba el aumento de riesgo de accidentes cardiovasculares graves con rofecoxib respecto a placebo $^{15}$.

La utilización de nuevos fármacos representa un porcentaje bajo de la prescripción en términos absolutos, esto se debe a que en este estudio sólo se ha seguido cada nuevo fármaco durante un año y el número de envases en ese período respecto al total de la prescripción supone un porcentaje pequeño. Por otro lado, se ha observado que los porcentajes de utilización son muy variables (entre el $0,3 \%$ y el $1,18 \%$ ), incluso entre centros del mismo tipo. Pero lo que es común para todos los centros es que concentran su prescripción de novedades en un número limitado de principios activos: 15 nuevos fármacos suponen del 80 al $90 \%$ del total de la prescripción de novedades.

Según el análisis multivariante realizado tras ajustar por diferentes variables aquellos centros en los que el gasto farmacéutico por paciente es mayor tienden a prescribir más novedades. Aunque podría pensarse que las novedades son más caras y por tanto podrían justificar mayor gasto ${ }^{3,16}$, este hecho no es claro, puesto que no todos los productos más nuevos superan en precio a los anteriores. Existen otros muchos factores que influyen en el gasto farmacéutico ${ }^{17,18}$ y consideramos que la mayor innovación en fármacos es más una consecuencia que una causa del gasto por paciente.

Al igual que en otros estudios ${ }^{9,12,19}$ parece haber una cierta relación entre mayor número de consultas por facultativo y la introducción de más novedades. Esto podría explicarse porque una mayor presión asistencial provoca falta de tiempo para la búsqueda de información objetiva sobre los nuevos fármacos. En estos casos, la mayoría de la información se obtiene de la industria farmacéutica que se inclina más por la promoción del medicamento que por la información científica ${ }^{2}$. 
La relación con el porcentaje de pensionistas es negativa, de manera que los centros con mayor proporción de pensionistas parecen tender a una menor prescripción de novedades. Esto también se describió en el estudio de Tamblyn et al ${ }^{12}$ y la razón podría deberse a que los ancianos presentan mayor riesgo de sufrir reacciones adversas a fármacos o interacciones farmacológicas, lo que no haría recomendable utilizar los medicamentos comercializados más recientemente, cuyos efectos son en gran parte desconocidos en este tipo de población.

Entre las limitaciones del estudio cabe destacar que se ha utilizado el número de envases, unidad que está siendo reemplazada en los estudios de utilización de medicamentos por el número de Dosis Diarias Definidas (DDD), ya que éste es un parámetro estándar que puede transformarse en Dosis/1.000 habitantes/día y permite realizar comparaciones entre países y períodos de tiempo diferentes. En nuestro caso no fue posible por limitaciones del programa informático. Por otra parte, para los nuevos fármacos no siempre está definida la DDD. No obstante, se considera que esta limitación no invalida los resultados del estudio.

El estudio presentado ha puesto de manifiesto el amplio uso de fármacos nuevos en el área de salud estudiada, no siempre justificado por la mejora en el potencial terapéutico. Se observa una gran variabilidad en el porcentaje de uso de novedades entre centros, encontrando una asociación positiva de esta variable con el número de consultas por facultativo y con el gasto farmacéutico por persona protegida, y negativa con el porcentaje de pensionistas entre la población atendida.

\section{BIBLIOGRAFÍA}

1. Prosser H, Walley T. New drug uptake: qualitative comparison of high and low prescribing GP's attitudes and approach. Fam Pract 2003;20:583-91.
2. Prescripción de nuevos medicamentos en Atención Primaria. Bol Ter Andal 2001;17(5).

3. Changing patterns of pharmaceutical innovation [informe en Internet]. Washington: NIHCM foundation; 2002 [consultado 24 de junio de 2004]. Disponible en: http://www.nihcm.org/innovations.pdf

4. Viejas moléculas bajo nueva apariencia ¿qué hay de nuevo? INFAC 2003;11(8):38-40.

5. Prescribing new drugs in general practice. MeReC Bull 1998;9(6):21-4.

6. Martín RM. When to use a new drug? Aust Prescr 1998;21:67-9.

7. Inman W, Pearce G. Prescribe profile and postmarketing surveillance. Lancet 1993;342:658-61.

8. McGavock H, Webb CH, Johnston GD, Milligan E. Market penetration of new drugs in one United Kingdom region: implications for general practitioners and administrators. BMJ 1993;307:1118-20.

9. Jones MI, Greenfield SM, Bradley CP. Prescribing new drugs: qualitative study of influences on consultants and general practitioners. BMJ 2001;323:378-81.

10. Cuesta MT, Martínez M. Nuevos principios activos: Revisión 2001 ( $2^{\mathrm{a}}$ parte). Inf Ter Sist Nac Salud 2002;26:48-60.

11. Peay MY, Peay ER. The role of commercial sources in the adoption of a new drug. Soc Sci Med 1988;26:1183-9.

12. Tamblyn R, McLeod P, Hanley JA, Girard N, Hurley J. Physician and practice characteristics associated with the early utilization of new prescription drugs. Med Care 2003;41:895-908.

13. García MA, Cabeza J, Rodríguez M, Alegre E, Rabadán A. Adopción de los nuevos medicamentos por los médicos prescriptores. El médico innovador. Aten Primaria 2000;25:22-8.

14. Díaz G, Palmeiro G, Núñez E, Casado I. Opinión de los médicos de Atención Primaria de Ourense sobre algunos aspectos de su prescripción farmacéutica. Rev Esp Salud Publica 2001;75:361-74.

15. Agencia Española de Medicamentos y Productos Sanitarios. Nota de seguridad relativa a la suspensión de comercialización de rofecoxib (Vioxx y Ceoxx). Nota informativa 2004/10. 16.- Disponible en http://www.agemed.es/Index.htm (acceso $18 \mathrm{de}$ marzo de 2005). 
16. Mason J, Freemantle N. The dilemma of new drugs. Are cost rising faster than effectiveness? Pharmacoeconomics 1998;13(6):653-7.

17. Caamaño F, Figueiras A, Gestal-Otero J.J.Condicionantes de la prescripción en Atención Primaria. Aten Primaria. 2001;27:43-8.
18. Carthy P, Harvey I, Brawn R, Watkins C. A study of factors associated with cost and variation in prescribing among GPs. Fam Pract 2000;17:36-41.

19. Strickland-Hodge B, Jepson MH. Identification and characterization of early and late prescribers in general practice. J R Soc Med 1982;75:341-5. 